\title{
Meridional Overturning Circulation and the Associated Heat and Freshwater Transports Observed by the OSNAP (Overturning in the Subpolar North Atlantic Program) Array from 2014 to 2016.
}

M. S. Lozier ${ }^{1 *}$, F. Li ${ }^{1 *}$, S. Bacon ${ }^{2}$, F. Bahr ${ }^{3}$, A. S. Bower ${ }^{3}$, S. A. Cunningham ${ }^{4}$, M. F. de Jong', L. de Steur ${ }^{5} \uparrow$, B. deYoung ${ }^{6}$, J. Fischer ${ }^{7}$, S. F. Gary ${ }^{4}$, B. J. W. Greenan ${ }^{8}$, N. P. Holliday ${ }^{2}$, A. Houk $^{9}$, L. Houpert ${ }^{4}$, M. E. Inall ${ }^{4,10}$, W. E. Johns ${ }^{9}$, C. Johnson ${ }^{4}$, J. Karstensen ${ }^{7}$, G. Koman 9 , I. A. Le Bras ${ }^{11}$, X. Lin ${ }^{12}$, N. Mackay ${ }^{13}+$, M. Oltmanns ${ }^{7}$, R. S. Pickart ${ }^{3}$, A. L. Ramsey ${ }^{3}$, D. Rayner ${ }^{2}$, F. Straneo $^{11}$, D. J. Torres ${ }^{3}$, I. Yashayaev ${ }^{8}$, J. Zhao ${ }^{3} \S$

${ }^{1}$ Division of Earth and Ocean Sciences, Duke University, Durham, NC, USA

${ }^{2}$ National Oceanography Centre, Southampton, UK

${ }^{3}$ Woods Hole Oceanographic Institution, Woods Hole, MA, USA

${ }^{4}$ Scottish Association for Marine Science, Oban, UK

${ }^{5}$ Royal Netherlands Institute for Sea Research and Utrecht University, Texel, Netherlands

${ }^{6}$ Department of Physics and Physical Oceanography, Memorial University, St. John's, Newfoundland, Canada

${ }^{7}$ GEOMAR Helmholtz Centre for Ocean Research Kiel, Kiel, Germany

${ }^{8}$ Bedford Institute of Oceanography, Dartmouth, Nova Scotia, Canada

${ }^{9}$ Department of Ocean Sciences, University of Miami, Miami, FL, USA

${ }^{10}$ College of Science and Engineering, University of Edinburgh, Edinburgh, UK

${ }^{11}$ Scripps Institution of Oceanography, UCSD, La Jolla, CA, USA

${ }^{12}$ Physical Oceanography Laboratory and Institute for Advanced Ocean Studies, Ocean University of China and Qingdao National Laboratory for Marine Science and Technology, Qingdao, China

${ }^{13}$ National Oceanography Centre, Liverpool, UK

$\dagger$ Present address: Norwegian Polar Institute, Tromsø, Norway $\ddagger$ Present address: Department of Geography, University of Exeter, Devon, UK $\S$ Present address: University of Maryland Center for Environmental Science, Cambridge, MD, USA

*Contact: M. Susan Lozier (mslozier@duke.edu); Feili Li (eili.li@duke.edu)

\section{File history}

June 2018

Data from the full OSNAP array for the first 21 months (31-Jul-2014 to 20-Apr-2016) have been used to produce the 30-day mean MOC, MHT and MFT time series across the section along with the gridded property fields. 


\section{Data management and policy}

The full OSNAP data policy and management plan is available online at: https://www.osnap.org/observations/data/ Before the use and publication of any OSNAP data, users are strongly encouraged to read the full OSNAP data policy. We draw your attention to the following excerpts from that policy:

- Any person making use of OSNAP observational data and/or numerical results must communicate with the responsible investigators at the start of the analysis and anticipate that the data collectors will be co-authors of published results.

- In cases where investigators choose not to be co-authors on publications that rely on their data, the parties responsible for collecting the data and the sponsoring funding agencies should be acknowledged, including reference to any relevant publications by the originating authors describing the data sets and a reference to the data set itself using its DOI.

- OSNAP data are intended for scholarly use by the academic and scientific community, with the express understanding that any such use will properly acknowledge the originating investigator.

The project scientists would appreciate it if you would use the data DOI (10.7924/r4z60gf0f) and add the following acknowledgement to any publication that use this data:

"OSNAP data were collected and made freely available by the OSNAP (Overturning in the Subpolar North Atlantic Program) project and all the national programs that contribute to it (www.o-snap.org)."

\section{Calculation methods}

Details of the calculation methods are given in the following publications:

[1] Lozier, M. S., Co-authors (2019): A sea change in our view of overturning in the subpolar North Atlantic. Science, doi:10.1126/science.aau6592.

[2] Li, F., M. S. Lozier, W. E. Johns (2017): Calculating the meridional volume, heat, and freshwater transports from an observing system in the subpolar North Atlantic: Observing System Simulation Experiment. J. Atmos. Oceanic Technol., 34, 1483-1500. doi:10.1175/JTECH-D-16-0247.1. 


\section{Description of the data}

Data in this collection are in netCDF format, including the following two separate files.

(a) MOC, MHT and MFT time series with uncertainty estimates

The file "OSNAP_Transports_201408_201604_2018.nc" contains 30-day mean estimates of the meridional overturning circulation and its associated heat and freshwater transports across the full OSNAP array for the period 31-Jul-2014 to 20-Apr-2016 (Table 1).

\begin{tabular}{ll}
\hline Data variable & Content \\
\hline TIME & Start date of each 30-day period [days since 1950-01-01] \\
MOC_SIGMA & Maximum of the overturning streamfunction in $\sigma_{\theta}$ coordinates [Sv] \\
MOC_SIGMA_ERR & Uncertainty for MOC_SIGMA [Sv] \\
MOC_Z & Maximum of the overturning streamfunction in z coordinates [Sv] \\
MOC_Z_ERR & Uncertainty for MOC_Z [Sv] \\
MHT & Total heat transport [PW] \\
MHT_ERR & Uncertainty for MHT [PW] \\
MFT & Total equivalent freshwater transport relative to the section-mean salinity [Sv] \\
MFT_ERR & Uncertainty for MFT [Sv] \\
\hline
\end{tabular}

Table 1. File contents for the time series product.

(b) Gridded temperature and salinity fields

The file "OSNAP_Gridded_TS_201408_201604_2018.nc” contains 30-day mean temperature and salinity fields on the OSNAP section for the period 31-Jul-2014 to 20-Apr-2016 (Table 2). 


\begin{tabular}{ll}
\hline Data variable & Content \\
\hline TIME & Start date of each 30-day period [days since 1950-01-01] \\
LONGITUDE & Longitude of the OSNAP grid [deg East] \\
LATITUDE & Latitude of the OSNAP grid [deg North] \\
DEPTH & Depth level of the OSNAP grid [m] \\
TEMP & Gridded temperature fields [deg C] \\
PSAL & Gridded practical salinity fields \\
\hline
\end{tabular}

Table 2. File contents for the gridded product. 